\title{
Nonlinear Pulse Propagation
}

\author{
Jeffrey Rauch
}

\begin{abstract}
This talk gives a brief review of some recent progress in the asymptotic analysis of short pulse solutions of nonlinear hyperbolic partial differential equations. This includes descriptions on the scales of geometric optics and diffractive geometric optics, and also studies of special situations where pulses passing through focal points can be analysed.
\end{abstract}

\section{Linear plane waves.}

Suppose that $L$ is an $N \times N$ system of partial differential operators

$$
L(\partial)=\partial_{t}+\sum_{i=1}^{d} A_{j} \partial_{x_{j}} .
$$

Here the spacetime variable is

$$
y=(t, x) \in \mathbb{R}^{1+d} \quad \text { with dual variables } \quad \eta=(\tau, \xi) .
$$

$L$ is assumed to be a symmetric hyperbolic operator in the sense that the $N \times N$ matrices $A_{j}$ are hermitian symmetric.

If $f: \mathbb{R} \rightarrow C^{N}$ is smooth then the chain rule yields

$$
L f(y \cdot \eta)=L(\eta) f^{\prime}(y \cdot \eta) .
$$

Thus $L(f(y \cdot \eta))=0$ when $f$ takes values in the nullspace of $L(\eta)$.

In this way the characteristic variety, the set of $\eta$ so that this nullspace is nontrivial, makes an appearance. For $\eta \in C h a r L$ one has the orthogonal decomposition

$$
\mathbb{C}^{N}=\operatorname{ker} L(\eta) \oplus_{\perp} \operatorname{range} L(\eta) .
$$

Introduce

$$
\pi(\eta)=\text { ortogonal projection onto } \operatorname{ker} L(\eta) .
$$

Then $u=f(y . \eta)$ is a plane wave solution of $L u=0$ when $\eta \in C$ har $L$ and $f$ satisfies the polarization

$$
\pi(\eta) f=f .
$$




\section{Plane pulses and group velocity.}

If in addition,

$$
f(s) \rightarrow 0 \quad \text { as } \quad s \rightarrow \pm \infty
$$

then the family of solutions

$$
u^{\epsilon}:=f\left(\frac{y \cdot \eta}{\epsilon}\right)
$$

describe pulses with planar wave fronts. If $f$ has compact support, then the pulse $u^{\epsilon}$ is supported in an $O(\epsilon)$ neighborhood of the hyperplane $y \cdot \eta=0$. The pulse cross section is given by the function $f(s / \epsilon)$. The function $f$ is called the profile of this pulse family.

At $t=0$ (resp. $t=1$ ) the pulse is supported near the planes $x . \xi=0$ (resp. $x . \xi=\tau)$.

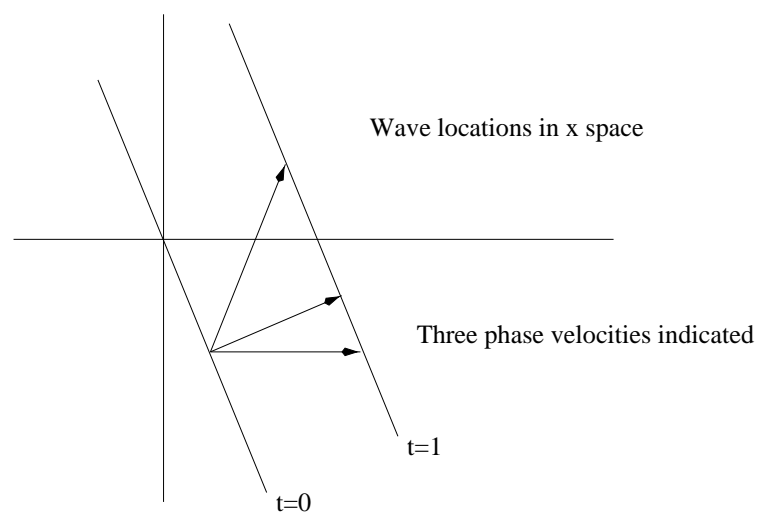

The phase

$$
\tau t+\xi . x=(x-\mathbf{v} t) \cdot \xi
$$

for any velocity vector $\mathbf{v}$ satisfying

$$
\mathbf{v} \cdot \xi=-\tau .
$$

For any such $\mathbf{v}$, the pulse family is given by

$$
f^{\epsilon}(x-\mathbf{v} t), \quad f^{\epsilon}(s):=f(s / \epsilon) .
$$

The pulse family can be viewed as moving with any one of these phase velocities. Three such velocities are sketched in the figure above. The phase velocity is not uniquely determined. This nonuniqueness is usually removed by choosing the unique $\mathbf{v}$ so that $\mathbf{v} \perp\{x . \xi=0\}$. This introduction of the euclidean scalar product in $x$ space is not natural. For example, when studying the operator

$$
u_{t t}=2 u_{x_{1} x_{1}}+u_{x_{2} x_{2}}
$$

there is a more natural scalar product. The velocity orthogonal in the natural scalar product is the right one and the velocity based on the euclidean convention is irrelevant. Summary. In dimensions $d>1$ the phase velocity is not well defined.

In contrast, the group velocity is well defined at smooth points $(y, \eta)$ of the characteristic variety. To see this, denote by $\mathcal{V} \subset T_{y}^{*}$ the characteristic variety at 
$y$. Since the operator $L$ is hyperbolic, $\mathcal{V}$ is a conic codimension one real algebraic subvariety of the cotangent space $T_{y}^{*}$. At smooth points the conormal variety $N^{*}(\mathcal{V})$ is therefore a one dimension subspace of $T_{y}^{* *} \equiv T_{y} \equiv \mathbb{R}^{1+d}$. Thus the line $N^{*}(\mathcal{V})=$ $\mathbb{R}(a, \mathbf{b})$ has a well defined velocity $\mathbf{v}=\mathbf{b} / a$ which is, by definition, the group velocity.

If the characteristic variety is given locally by the equation $\tau=\tau(t, x, \xi)$ then this recipe reduces to the standard physics book formula

$$
\text { group velocity }:=\mathbf{v}(t, x, \xi)=-\nabla_{\xi} \tau(t, x, \xi) .
$$

The vector field $\partial_{t}+\mathbf{v} \cdot \partial_{x}$ is then equal to the space time part of the hamilton vector field with hamiltonian $\tau-\tau(t, x, \xi)$.

Since $\tau$ is homogeneous of degree 1 in $\xi$, the Euler homogeneity relation implies that

$$
\xi . \nabla_{\xi} \tau(t, x, \xi)=\tau(t, x, \xi) .
$$

This implies that the group velocity satisfies $\mathbf{v} . \xi=-\tau$, the equation defining phase velocities. The group velocity is the right phase velocity.

At points of the characteristic variety which are not smooth, neither the group nor the phase velocity is well defined.

The group velocity enters the approximations of geometric optics though the algebraic identity (see [DJMR])

$$
\pi(y, \eta) L(y, \partial) \pi(y, \eta)=\pi(y, \eta)\left[\left(\partial_{t}+\mathbf{v}(y, \eta) . \partial_{x}\right)+\sum_{j} A_{j}(y) \frac{\partial \pi(y, \eta)}{\partial x_{j}}\right] \pi(y, \eta)
$$

In our work the projectors $\pi$ replace the left and right eigenvectors that one finds in traditional treatments.

\section{Wave trains versus pulses.}
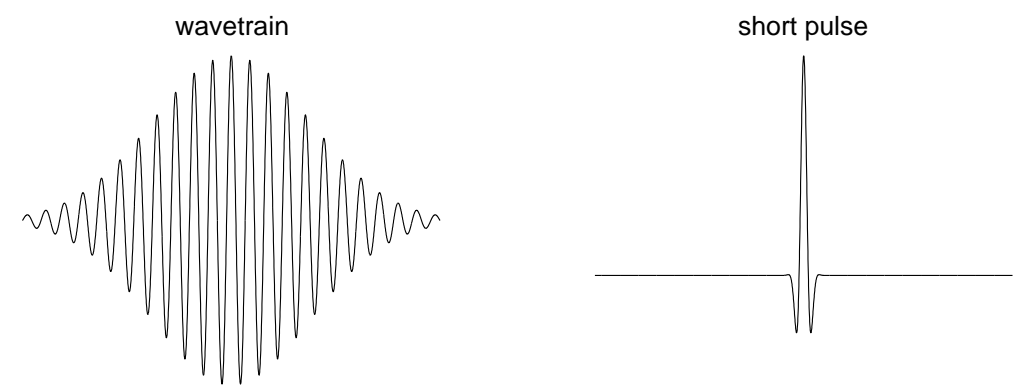

The geometric optics approximations which are most familiar concern the short wavelength limit of wave trains. The usual methods yield equations for the envelope and go under the name of the slowly varying envelope approximation (svea) in science journals. A rule of thumb is that the amplitude should not change more than $10 \%$ per wavelength. This criterion is just barely violated in the figure at the left. The rule of thumb suggest that one must have about twenty wavelengths per pulse length before the svea is appropriate.

For much shorter pulses like the one on the right in the figure the svea is clearly inapplicable (see [R], [AR1]). 
Typical analytic expressions for the waveforms above are

$$
\begin{array}{lclr}
\text { wavetrain : } & a(x) e^{i x_{1} / \epsilon} & \text { with Fourier transform } & \hat{a}(\xi-(1 / \epsilon), 0)) \\
\text { pulse : } & a\left(x_{1} / \epsilon\right) b\left(x^{\prime}\right) & \text { with Fourier transform } & \epsilon \hat{a}\left(\epsilon \xi_{1}\right) \hat{b}\left(\xi^{\prime}\right) .
\end{array}
$$

The Fourier transform of the wave train is localized near $(1 / \epsilon, 0)$ which is called the carrier frequency in applications.

The Fourier transform of the pulse is spread over a box of dimensions $1 / \epsilon \times 1$ in $\left(\xi_{1}, \xi^{\prime}\right)$ space. There is no carrier frequency. There is no exponential prefactor which renders the quotient slowly varying.

The approximations take the form

$$
\begin{array}{llll}
\text { wave train : } & U(y, \phi(y) / \epsilon) & \text { with } & U(y, \theta) \text { periodic in } \theta \\
\text { pulse : } & U(y, \phi(y) / \epsilon) & \text { with } & U(y, z) \rightarrow 0 \text { as } z \rightarrow 0 .
\end{array}
$$

In both cases the phase function $\phi$ satisfies an eikonal equation.

In the latter case the function $U(y, \cdot)$ represents the profile of the pulse. The surfaces $\phi=$ const are wavefronts. The pulse approximation can be called the slowly varying profile approximation since the profiles vary on the scale $O(1)$ which is much longer than the wavelength $\epsilon$

\section{Overview}

The recent work that I will mention can be organized into four categories.

I. Geometric optics scaling before focusing, (Alterman-Rauch).

II. Focus crossing for radial $\square_{1+3}$, (Carles-Rauch).

III. Diffractive optics scaling, (Alterman-Rauch).

IV. Solutions with large spectrum, (Barrailh-Lannes).

Preprints of the articles for the first two are available from my home page. The third should make it shortly.

Overview I. The results concern solutions $u^{\epsilon}$ of

$$
L(y, \partial) u^{\epsilon}+\Phi\left(u^{\epsilon}\right)=0, \quad u^{\epsilon}(0, x)=f\left(x, \frac{\phi(0, x)}{\epsilon}\right), \quad f(x, z)=\pi(\eta) f .
$$

which are of size $O(1)$ so the nonlinear term is of size $O(1)$ and its effects are felt at time $t=O(1)$.

The analysis is carried out for variable coefficient operators and for pulses which may have curved wavefronts which can degenerate at focal points. The analysis is valid before the focal points.

The equations defining the approximate pulse solutions are similar to the equations of geometric optics which govern wave trains.

Sketches of the wavefronts are presented in the figure below. The figure on the left evokes an expanding wavefront. The amplitudes will decrease correspondingly. The figure on the right evokes a planar pulse with transverse dimension $O(1)$, parallel rays and no geometric decay. 

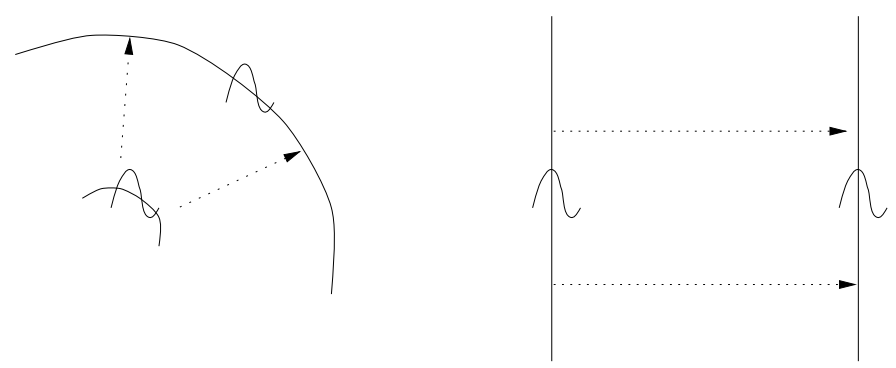

Overview II. The passage of a pulse through a focal point has a chance of being easier to analyse than the corresponding problem for wave trains, because the pulse spends only $O(\epsilon)$ units of time near the focal point. This simple idea has been born out by a number of successfully analysed problems. We hope that this is just the beginning.

The results that have been proved concern radial solutions of equations of the form $\square_{1+3} u+f\left(\nabla_{t, r} u\right)=0$. Begin by recalling that in the linear case, $f=0$, a pulse emerges from a spherical focus with its amplitude multiplied by -1 . The same is true of all incoming spherical solutions of the linear wave equation, not just pulses.

To avoid long lists of possibilities I discuss here only

$$
\begin{gathered}
f=a u_{t}\left|u_{t}\right|^{h-1}, \quad h=\text { order of nonlinearity }>1, \quad a \in \mathbb{C} . \\
\text { When }\left\{\begin{array}{ccc}
a>0 & \text { the problem is } & \text { dissipative } \\
a<0 & \text { it is } & \text { accretive } \\
a \in i \mathbb{R} & \text { it is } & \text { conservative. }
\end{array}\right.
\end{gathered}
$$

Consider pulses focusing along the incoming light cone $r=1-t$. For $t<1$ the description of pulses with $\nabla u=O(1)$ is given by the geometric optics from the previous subsection. As the pulses approach the origin the size of the gradient grows so the behavior of the nonlinear function at infinity becomes important.

There is a critical power $h_{c}$.

In the subcritical case $1<h<h_{c}$, classical solutions exist beyond the focus provided the initial profiles are sufficiently small (independent of $\epsilon$ ). There is a region of size $\epsilon^{\alpha}$ with $0<\alpha<1$ which is large compared to the wavelength, small compared to 1 , and centered at the focal point so that in that region the exact solution is well approximated by a wavelength $\epsilon$ pulse solution of the linear wave equation. Beyond that region nonlinear geometric optics takes over again. Crossing the focus multiplies the pulse by -1 as in the linear case.

\section{RADIAL 1+3 D SEMILINEAR WAVE EQN}

subcritical yields linear passage

critical yields nl scattering

supercritical dissip yields absorbtion

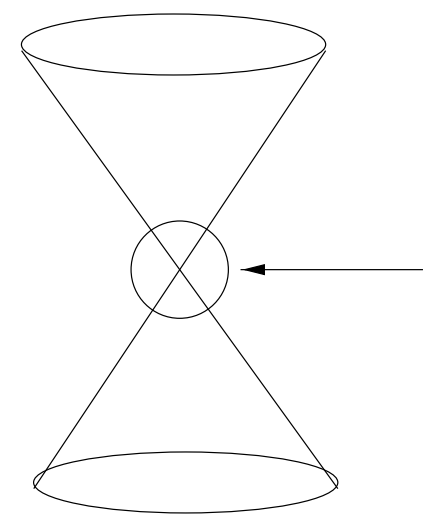


In the supercritical case $h_{c}<h$ with $a>0$ so that the nonlinearity is dissipative, the pulse is absorbed at the focus in the sense that the outgoing wave has gradient $o(1)$ as $\epsilon \rightarrow 0$.

The most interesting case is the critical case for which the crossing of the focus is described by a nonlinear scattering operator. For more details and more cases including propagations which are linear before the focus and can be subcritical, critical, or supercritical at the focus, invite Remi Carles.

Overview III. These results are the ones which I will describe in more detail in part because the authors are from across the Atlantic and therefore less easy to hear. The principal part of the differential equation must have constant coefficients and the phase is chosen linear. Then the wavefronts are planar and the rays are parallel so stay close together for long time scale $t=O(1 / \epsilon)$ of interest. The case of nearly planar wave trains is treated in the thesis of Eric Dumas [Du]. The approximate solutions have the form

$$
u_{\text {approx }}^{\epsilon}=\epsilon^{p} U_{0}\left(\epsilon t, t, x, \frac{\tau t+\xi . x}{\epsilon}\right), \quad \lim _{|z| \rightarrow \infty} U_{0}(T, t, x, z)=0 .
$$

The power $p$ satisfies

$$
p=1 /(J-1)
$$

where the leading Taylor polynomial at $u=0$ of the nonlinear term is of order $J$. Then, the nonlinear term is of size $\epsilon^{p J}$ and $p J=p+1$. The geometric effects of diffraction for planar wavefronts take place at times $t=O(1 / \epsilon)$ (see [DJMR]) so the accumulated effect of the nonlinear term is crudely estimated as

$$
\frac{1}{\epsilon} \epsilon^{p+1}=\epsilon^{p}
$$

The amplitude is tuned so that time of nonlinear interaction is comparable to the diffraction time.

The slowly varying profile $U_{0}$ is polarized as usual, $\pi(\eta) U=U$, and is determined from its initial data by the pair of evolution equations

$$
\left(\partial_{t}+\mathbf{v} . \partial_{x}\right) U_{0}=0, \quad \partial_{T z} U_{0}+\frac{1}{2} \tau_{\xi \xi}^{\prime \prime}\left(\partial_{x}, \partial_{x}\right) U_{0}+\pi(\eta) \partial_{z} \Phi_{J}\left(U_{0}\right)=0
$$

where $\Phi_{J}$ is the leading Taylor polynomial of $\Phi$. This evolution equation for which $T=0$ is characteristic, is the pulse version of the nonlinear Schrödinger equation.

To see that the last equation is in fact a nice evolution equation write it formally as

$$
\partial_{T} U_{0}+\frac{1}{2} \partial_{z}{ }^{-1} \tau_{\xi \xi}^{\prime \prime}\left(\partial_{x}, \partial_{x}\right) U_{0}+\pi(\eta) \Phi_{J}\left(U_{0}\right)=0
$$

The operator $\partial_{z}^{-1} \tau_{\xi \xi}^{\prime \prime}\left(\partial_{x}, \partial_{x}\right)$ is antisymmetric on the $H^{s}$ which for $s$ large are invariant under $\Phi_{J}$ and Picard's method yields local solvability. One must be a little careful to take care of the translation invariance required by the first of the equations.

Overview IV. These results are on the scale of geometric optics and diffractive optics and meld the pulse like solutions, whose spectrum is very broad, with other 
solutions with rich spectrum constructed in the thesis of D. Lannes. The authors have introduced a variety of efficient analytic techniques. One of the principal interests of these results is to quantify the interactions between these waves. One result is that since pulses occupy such a small region of space time they tend to interact only for times $O(\epsilon)$ which tends to make the interaction negligible. For more details invite the authors from Bordeaux.

\section{Ideas from the proof of III. 1. The singular system.}

A handle on the exact solution is obtained by expressing that solution in the form

$$
u^{\epsilon}(t, x)=\epsilon^{p} \mathcal{V}\left(\epsilon, t, x, \frac{x . \xi}{\epsilon}\right)
$$

where $\mathcal{V}(\epsilon, t, x, \phi)$ solves the singular initial value problem

$$
L\left(\partial_{t}, \partial_{x}+\frac{\xi}{\epsilon} \partial_{\phi}\right) \epsilon^{p} \mathcal{V}+\Phi\left(\epsilon^{p} \mathcal{V}\right)=0=0, \quad \mathcal{V}(\epsilon, 0, x, \phi)=f(x, \phi)
$$

The singular part is

$$
\frac{1}{\epsilon} \sum_{j} \xi_{j} A_{j} \frac{\partial}{\partial \phi}
$$

which is an antisymmetric operator on all $H^{s}\left(\mathbb{R}_{x, \phi}^{d+1}\right)$. The solutions are nicely bounded in these spaces for $t=O(1 / \epsilon)$ since the nonlinear term is $O\left(\epsilon \cdot \epsilon^{p}\right)$. The time derivates of solutions are $O(1 / \epsilon)$.

Theorem. If the profile equations for $U$ are solvable for $0 \leq T \leq \underline{T}$, then the approximate solution

$$
u_{\text {approx }}^{\epsilon}=\epsilon^{p} U_{0}\left(\epsilon t, t, x, \frac{\tau t+\xi \cdot x}{\epsilon}\right) .
$$

and the exact solution are close for $t=O(1 / \epsilon)$ in the sense that for $s \in \mathbb{R}$

$$
\sup _{0 \leq t \leq \underline{T} / \epsilon}\left\|\mathcal{V}(t, x, \phi)-U_{0}\left(\epsilon t, t, x, \frac{\tau t}{\epsilon}+\phi\right)\right\|_{H^{s}\left(\mathbb{R}_{x, \phi}^{d+1}\right)}=O\left(\epsilon^{\min \{p, 1 / 5\}}\right)
$$

The $1 / 5$ in the exponent is surely not sharp. Our current best guess at the sharp value is 1 .

This singular system is reminiscent of the strong coriolis term in models of geophysics and in the incompressible limit in fluid mechanics. In fact, the asymptotic analysis in a variety of fluids applications (see Schochet, Grenier, Masmoudi, Klainerman, Majda, Galagher, Colin, Ghidaglia, Lannes, ....) is parallel too and quite close in spirit and in detail to what is done in the work on nonlinear geometric optics. Schochet recognized this from the outset. It is not unreasonable to hope for a grand unification. 


\section{Ideas from the proof III. 2. Small divisors and infrared cutoff.}

As in virtually all problems of this type, if the approximate solution is plugged into the differential equation, the residual is too large. In this case the residual is $O\left(\epsilon^{p+1}\right)$ which on a time interval $O(1 / \epsilon)$ is expected to accumulate to $O\left(\epsilon^{p}\right)$ which is the same size as the exact and the approximate solutions.

To overcome this, attempt to construct correctors

$$
U(\epsilon, T, y, z)=U_{0}(T, y, z)+\epsilon U_{1}(T, y, z)+\epsilon^{2} U_{2}(T, y, z)
$$

Then $u_{\text {approx }}^{\epsilon}$ would be an exact solution if

$$
L\left(\left(\epsilon \partial_{T}, 0\right)+\partial_{y}+\frac{\eta}{\epsilon} \partial_{z}\right) \epsilon^{p} U(\epsilon, T, y, z)+\Phi\left(\epsilon^{p} U(\epsilon, T, y, z)\right)=0 .
$$

What we do is expand the left hand side in powers of $\epsilon$ and try to kill as many terms as possible. A somewhat clever computation leads to the profile equations for $U_{0}$ which have already been cited. A key is the algebraic identity cited at the end of section 3 .

For the first corrector $U_{1}$ one finds

$$
\pi(\eta) U_{1}=0, \quad \text { and } \quad(I-\pi(\eta)) \partial_{z} U_{1}=-Q(\eta) L\left(\partial_{y}\right) U_{0} .
$$

Here $Q(\eta)$ is the partial inverse of the singular hermitian matrix $L(\eta)$. These equations are not in general solvable. The solution if it exists is given by

$$
\left(\mathcal{F} U_{1}\right)(t, x, \zeta)=\frac{-1}{i \zeta} Q(\eta) L\left(\partial_{y}\right)\left(\mathcal{F} U_{0}\right)(t, x, \zeta) .
$$

For the right hand side to make sense requires that

$$
Q(\eta) L\left(\partial_{y}\right)\left(\mathcal{F} U_{0}\right)(t, x, 0)=0 \quad\left(\text { l.h.s. }=\int Q L U_{0}(t, x, z) d z\right) .
$$

This condition of vanishing mean is neither generic, nor natural in the physical applications. The formula for $(I-\pi(\eta)) U_{1}$ has zero (resp. small) divisors at $\zeta=0$ (resp. $\zeta \approx 0$ ). These represent small divisor problems in a setting of continuous spectrum.

To overcome this difficulty we introduce an infrared cutoff in the equation defining $U$. Choose a cutoff function $\chi(\zeta) \in C^{\infty}(\mathbb{R})$ which vanishes on $|\zeta| \leq 1$ and is identically equal to 1 for $|\zeta| \geq 2$. Define

$$
\chi^{\delta}\left(D_{z}\right)=\mathcal{F}^{*} \chi(\zeta / \delta) \mathcal{F},
$$

where $\mathcal{F}$ denotes the Fourier Transform in $z$. Seek

$$
U^{\delta}(\epsilon, T, y, z)=U_{0}^{\delta}(T, y, z)+\epsilon U_{1}^{\delta}(T, y, z)+\epsilon^{2} U_{2}^{\delta}(T, y, z)
$$

as an approximate solution of the cutoff equation

$$
L\left(\left(\epsilon \partial_{T}, 0\right)+\partial_{y}+\frac{\eta}{\epsilon} \partial_{z}\right) \epsilon^{p} U^{\delta}(\epsilon, T, y, z)+\chi^{\delta}\left(D_{z}\right) \Phi\left(\epsilon^{p} U^{\delta}(\epsilon, T, y, z)\right)=O\left(\epsilon^{2 p+1}\right) .
$$

Having removed the frequencies near 0 , this can be achieved. The correctors $U_{j}^{\delta}$ grow like $1 / \delta^{j}$ as $\delta \rightarrow 0$.

The proof proceeds by estimating errors, then choosing $\delta=\epsilon^{2 / 5}$ to optimize. 


\section{Ideas from the proof III. 3. Cutoff errors and the Wiener algebra.}

A key element in the proof is to estimate the cutoff error

$$
\left(1-\chi^{\delta}\left(D_{z}\right)\right) U_{0}(T, y, z) .
$$

We know that $\left(\partial_{t}+\mathbf{v} \cdot \partial_{x}\right) U_{0}=0$ so write

$$
U_{0}=\mathrm{U}_{0}(T, x-\mathbf{v} t, z)
$$

and we need to estimate

$$
\left(1-\chi^{\delta}\left(D_{z}\right)\right) \mathrm{U}_{0}(T, x, z) .
$$

If we know that $\mathrm{U}_{0} \in \cap_{s} H^{s}\left(\mathbb{R}_{x, z}^{d+1}\right)$ this error is $O(\sqrt{\delta})$ as one proves by applying the Cauchy-Schwartz inequality to the integral over $|\zeta|<\delta$.

In contrast to the nonlinear Schrödinger equation from the wave train case, the equation satisfied by $\mathrm{U}_{0}$ does not leave the Schwartz space $\mathcal{S}\left(\mathbb{R}^{d+1}\right)$ invariant. To improve the error estimate from $O(\sqrt{\delta})$ to $O(\delta)$ one needs $L^{\infty}$ control on the Fourier Transform of $\mathrm{U}_{0}$ Normally that would be done by proving decay estimates for $\mathrm{U}_{0}$. The decay of $\mathrm{U}_{0}$ is both subtle and not rapid. Instead, we work directly in Fourier.

The equation satisfied by $\mathrm{U}_{0}$ is semilinear with constant coefficient leading part and polynomial nonlinear term. The linear propagator is the Fourier multiplier

$$
e^{\frac{i t \tau^{\prime \prime}(\xi, \xi)}{2 \zeta}}
$$

which has modulus one. It defines an isometry on the Sobolev spaces $H^{s}$ and also on the Wiener algebra defined by $\hat{F} \in L^{1}$ and also the spaces of distributions defined by $\hat{F} \in L^{\infty}$. Working directly in Fourier we are able to bound

$$
\langle\xi, \zeta\rangle^{s} \hat{\mathrm{U}}_{0}(T, \xi, \zeta) \in L^{\infty}\left(\mathbb{R}_{\xi, \zeta}^{d+1}\right)
$$

for all $s$.

That $\left(\mathcal{F} \mathrm{U}_{0}\right) \in L^{1}$ is a natural condition is easy to appreciate. The linear propagation is an isometry, and the space is an algebra so invariant under the polynomial linearity. From there, it is not hard to show that the space defined by $\langle\xi, \zeta\rangle^{s} \hat{\mathrm{U}}_{0} \in L^{\infty}\left(\mathbb{R}^{d+1}\right)$ is propagated by the profile equation.

\section{References}

[1] D. Alterman, Diffractive nonlinear geometric optics for short pulses, Ph.D. Thesis, University of Michigan, May 1999.

[2] D. Alterman and J. Rauch, Diffractive short pulse asymptotics for nonlinear wave equations, Phys. Lett. A. 264(5)2000, pp. 390-395.

[3] D. Alterman and J. Rauch, Nonlinear geometric optics for short pulses, Journal of Differential Equations, to appear. 
[4] D. Alterman and J. Rauch, The linear diffractive pulse equation, Methods and Applications of Analysis 7(2001), to appear.

[5] Baraill and D. Lannes, In preparation.

[6] R. Carles, Geometric optics with caustic crossing for some nonlinear Schrödinger equations, Indiana Univ. Math. J. 49(2000) 475-551.

[7] R. Carles, Focusing on a line for nonlinear Schrödinger equations in $\mathbb{R}^{2}$, Asymptotic Analysis 24(2000) 255-276.

[8] R. Carles and J. Rauch, Focusing of spherical nonlinear pulses in $\mathbb{R}^{1+3}$, Proc. AMS (2001), to appear

[9] R. Carles and J. Rauch, Absorption d'impulsions non-linéaires radiales focalisantes dans $\mathbb{R}^{1+3}$, Note CRAS, to appear.

[10] R. Carles and J. Rauch, Diffusion d'impulsions non-linéaires radiales focalisantes dans $\mathbb{R}^{1+3}$, Note CRAS to appear.

[11] P. Donnat, J.-L. Joly, G. Métivier, and J. Rauch, Diffractive nonlinear geometric optics, Séminaire Equations aux Dérivées Partielles, Ecole Polytechnique, Paris, 1995-1996.

[12] E. Dumas, Univ. Rennes I Thesis, Fall 2000.

[13] J.-L. Joly, G. Métivier, and J. Rauch, Diffractive nonlinear geometric optics with rectification, Indiana Math. J. 47(1998) 1167-1241.

[14] J.-L. Joly, G. Métivier, and J. Rauch, Transparent nonlinear geometric optics and Maxwell-Bloch equations, J. Diff. Eq. 166(2000), 175-250.

[15] A. Majda, Nonlinear geometric optics for hyperbolic systems of conservation laws, Oscillation theory, computation, methods of compensated compactness, IMA Vol. Math. Appl. 2, Springer, New York, 1986, pp. 115-165.

[16] A. Majda and R. Rosales, Resonantly interacting weakly nonlinear hyperbolic waves I, Stud. Appl. Math., 71(1984), 149-179.

[17] J. E. Rothenberg, Space-time focusing: breakdown of the slowly varying envelope approximation in the self-focusing of femtosecond pulses, Optics Letters, 17(1992), 1340-1342.

[18] S. Schochet, Fast singular limits of hyperbolic partial differential equations, J. Diff. Eq. 114((1994), 474-512

[19] A. Yoshikawa, Solutions containing a large parameter of a quasi-linear hyperbolic system of equations and their nonlinear geometric optics approximation, Trans. A.M.S., 340(1993), 103-126. 
[20] A. Yoshikawa, Asymptotic expansions of the solutions to a class of quasilinear hyperbolic initial value problems, J. Math. Soc. Japan, (47)1995, $227-252$.

\section{Department of Mathematics}

EAST HALL

UNIVERSITY OF MICHIGAN

ANn ARBor MI, 48104, USA

rauch@umich.edu

www.math.lsa.umich.edu/ rauch 